\author{
L. Woltjer \\ European Southern Observatory \\ Karl-Schwarzschild-Strasse 2 \\ D-8046 Garching b. München
}

\title{
INTRODUCTION
}

In 1930 B. Schmidt invented the telescope that bears his name. Eleven years later, there were already two dozen Schmidt telescopes with primaries of $25 \mathrm{~cm}$ or more in diameter (Dimitroff and Baker 1945). An invention spreading so rapidly must have fulfilled a basic need - the need for a large field with good image quality over a substantial wavelength range. Here I shall briefly review the role of the large Schmidt telescopes in contemporary astronomy.

\section{SKY SURVEYS}

Perhaps the most important contributions made by Schmidt telescopes is that they have provided for the first time very deep maps of the whole sky down to stellar magnitude 20 and beyond. In table 1 the large scale deep surveys are listed. The Palomar Sky survey and the ESO Quick Blue Survey have been fully completed, the SRC Blue survey is nearly complete, and the others are in progress. The newer photographic emulsions have given significant gains in both resolution and limiting magnitude. As a consequence, the recent decision at Palomar to make a new survey of the northern sky is most welcome.

In addition to these large mapping surveys, also more specialized surveys have been undertaken at various observatories covering important parts of the sky: numerous surveys for blue stars (on the basis of U-B and/or U-V colour), near IR surveys, surveys with special colour filters for emission line objects, spectral surveys for the classification of stars or for the discovery of quasars, and surveys for variable stars, stars of large proper motion and asteroids. Surveys for highly polarized objects could also be of interest, but to date only a few experimental studies have been made. 
Table 1
D E E P
S K Y
$S$ U R V E Y S

$\begin{array}{lcccccc}\text { SURVEY } & \text { COLOUR } & \text { DEC } & \text { N } & \text { EMULSION } & \lambda & \text { m } \\ & & & & & & \\ \text { PALOMAR } & \text { BLUE } & >-33 & 935 & 103 a-0 & 3400-4800 & 21 \\ & \text { RED } & >-33 & 935 & 103 a-E & 6300-6600 & 20 \\ \text { ESO QUICK } & \text { BLUE } & <-18 & 606 & \text { IIa-O } & 3700-5000 & 21 \\ \text { ESO/SRC } & \text { BLUE } & <-17 & 606 & \text { IIIa-J } & 3800-5300 & 23 \\ & \text { RED } & <-18 & 606 & \text { IIIa-F } & 6300-6800 & 22 \\ & & & & & & \\ \text { UK SCHMIDT } & \text { BLUE } & -18-+3 & 288 & \text { IIIa-J } & 3800-5300 & 23 \\ \text { EQUATORIAL } & \text { RED } & -18-+3 & 288 & \text { IIIa-F } & 6300-6800 & 22\end{array}$

Note: $N=$ number of fields

$\lambda=$ estimated half power wavelength limits of the filter-plate combination

$m$ = limiting magnitude on the best plates

Before proceeding with a discussion of the scientific areas in which these surveys are of importance, it is necessary to consider for a moment the relative roles of schmidt telescopes and of large reflectors in the $4-\mathrm{m}$ class. Of course, as has been pointed out frequently, the Schmidt surveys have led to the discovery of many "interesting" objects that subsequently have been studied with large telescopes in spectroscopic and imaging modes. But for many survey type studies the better scale of large telescopes would also be preferable. 
As an example, in figure 1 a comparison is made between the images of the Sculptor dwarf irregular galaxy (Laustsen et al 1977) and its surroundings on the ESO cuick Blue Survey, the SCR blue (J) survey, the ESO red (F) survey, and on a IIIa-J plate taken with the ESO $3.6 \mathrm{~m}$ telescope. The greater depth of the IIIa-J in comparison with the IIa-O is very much in evidence, as is the superiority of the $3.6-\mathrm{m}$ image in comparison with that obtained with the schmidt

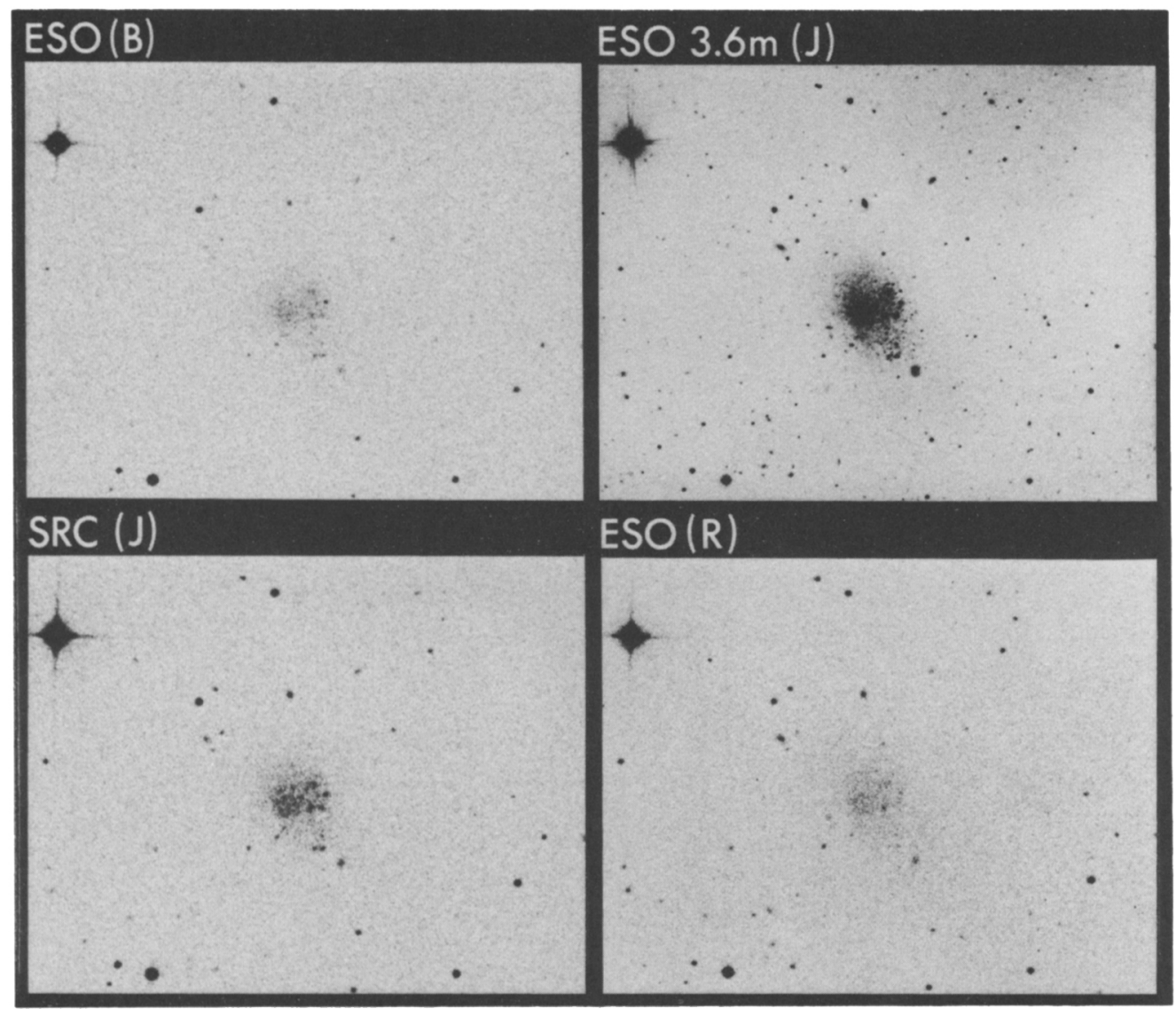

Figure 1: The Sculptor Dwarf Irregular Galaxy photographed with the ESO Schmidt in the Quick Blue Survey on IIa-O (ESO-B) and in the red survey on IIIa-F (ESO-R), with the UK Schmidt in the blue survey on IIIa-J (SRC-J) and with the ESO $3.6 \mathrm{~m}$ telescope on IIIa-J (ESO $3.6 \mathrm{~m}-\mathrm{J}$ ). The bright blue stars in the dwarf galaxy are clearly visible on the blue exposures. The surrounding foreground stars and background galaxies have on the average about equal images on the $\operatorname{SRC}(J)$ and ESO(R) plates. 
telescopes. While the bright blue stars of the dwarf galaxy are comparatively faint on the red survey, it is interesting to note that almost all field stars visible on the red survey plate are visible on the blue one and vice versa. However, many more field stars are visible on the $3.6-\mathrm{m}$ plates, and also the distinction between faint galaxies and stars is much clearer. Suppose then we have both a Schmidt and a large telescope available, what criterion should we apply to decide which of the two to use for a particular survey ?

A crude answer may be based on the following considerations. The field of a large telescope is usually of the order of a square degree. In a typical deep survey, several plates have to be taken per field, and with the pressure on large telescope time it is difficult to extend such a survey much beyond 10 square degrees. To learn something about the objects being surveyed, one should be at least able to determine their number with some accuracy - perhaps to within 20 \%. This would require 25 objects to be detected in the survey, corresponding to 2.5 objects per square degree or to a total number over the whole sky $\mathrm{N}=10^{5}$. While one may vary these numbers a bit, the conclusion is clear: if $N \gg 10^{5}$ it is reasonable to make a survey with a large telescope, but if $\mathrm{N} \ll 10^{5}$ this is impossible, and a large field schmidt is needed. Of course, this does not mean that one cannot use a Schmidt also in the former case, especially when no other telescope is available, but the results are likely to be less satisfactory if the objects are faint.

Another way of looking at the situation is to note that an all-sky survey in two colours with large telescopes would require about $10^{5}$ plates corresponding to about 25,000 dark nights or about 250 telescope years. With around 10 large telescopes in the world enyayed for 10 of the dark time in such a survey, it would take 250 years to complete it. With five of the world's large schmidts doing the same programme, a gain of a factor of $5 \times 30=150$ in time would be achieved - the factor of 30 arising from the larger field. As a result, such a survey could be completed in two years. If one were to add 3 spectral plates and 3 more colours per field, the time required would become four times larger. With a fúrther 20 plates for searches for variables and for astrometry, 3500 years would be needed with the large telescopes and 25 years with the five schmidts. What these figures show is that with large telescopes less than a few percent of the sky is likely to be imaged during the coming decades. On the other hand, though some selectivity is needed in Schmidt proyrammes, the present schmidt telescopes in the world appear to be sufficient to deal with much of what is needed during the medium term future.

\section{SCIENTIFIC PROGRAMMES WITH SCHMIDT TELESCOPES}

In table 2 are listed some typical objects for which the all-sky number $\mathrm{N} \leqslant 10^{5}$ and which consequently are in the prime domain of research with Schmidt telescopes. 
Table 2

TYPICAL OBJECTS IN SCHMIDT ASTRONOMY

\author{
GALAXIES $\quad B<16$ \\ DWARF GALAXIES \\ PECULIAR GALAXIES \\ QUASARS $\quad B<19$ \\ BL LAC \\ CLUSTERS OF GALAXIES \\ SUPERCLUSTERS \\ PECULIAR STARS $\quad B<15$ \\ VARIABLE STARS \\ LOW METALLICITY STARS \\ INTRINSICALLY FAINT STARS \\ $z<0.5$ \\ $z<1$
}

\author{
PLANETARIES \\ H II REGIONS \\ SUPERNOVA REMNANTS \\ DARK NEBULAE:
}

Relatively bright galaxies have been extensively searched for on the sky surveys by zwicky et al. (1968), by Vorontsov-Velyaminov and Arhipova (1968), by Nilson (1973) and by Lauberts (1982). The catalogues made by these authors include a total of about 50,000 galaxies down to (uncertain) photographic magnitudes of about 15.5 or angular diameters of about 1 arc minute. These catalogues have served as a rich material for further studies with larger telescopes and also for the study of the distribution of galaxies in space.

About 1800 dwarf galaxies - difficult to detect because of low surface brightness - have been found on the sky surveys by Tully (see van woerden 1980). Many of these have been subsequently studied by 21-cm radio techniques. Many "interacting", "peculiar" and "eruptive" galaxies have been found by Vorontsov-Velyaminov (1959), by Lauberts (1982), by Arp and Madore (unpublished), and by Zwicky (1971). Some of these have been studied in more detail also spectroscopically leading to concepts involving tidal interaction between galaxies and eruptive phenomena in the nuclei of galaxies. Seyfert galaxies and galaxies with active star formation have been found by Markarian (1969) on the basis of their blue continuum on objective prism plates.

In studies of the distribution of fainter galaxies on the Palomar survey, Abell (1958) and Zwicky and his associates (1968) found numerous clusters which have gained in importance with the discovery of much associated hot, $x$-ray emitting gas. It is now generally agreed that the distribution of clusters is not random, and this has led to the concept of superclusters as possibly the largest units in the universe.

Quasars were first discovered on the basis of the identification of radio sources. The subsequent discovery by Sandage (1965) that quasars without perceptible radio emission constitute a far more 
numerous class resulted from searches of Schmidt plates on the basis of $U-B$ and $U-V$ colours. Apart from the U-B excess which since that time has been found to be a good indicator for quasars with redshifts less than $z=2.2$, quasars have been found on the basis of the detection of emission lines in low dispersion objective prism plates taken with Schmidt telescopes. A large survey for U-b excess objects covering $1 / 4$ of the whole sky has recently been made by Schmidt and Green (1983). Apart from about a hundred quasars and Seyferts with B $<16$, more than a thousand white dwarfs, cataclysmic variables and other blue stars were discovered.

The number of quasars as a function of magnitude increases very steeply due to cosmic evolution (factor of eight per magnitude) and, as a consequence, small variations in photometric characteristics of plates may lead to large variations in the numbers found. Nevertheless, the very large incompleteness (factor of about 3 ) in the objective prism surveys with the 24 -inch Schmidt at Tololo as shown by comparison with data from the grism surveys with the 4-m telescope (Osmer 1980, Woltjer and Setti 1982) remains surprising.

The quasars provide a good example of the interplay between Schmidt telescopes and large reflectors. Virtually all information below $B=19$ comes from the former, while the latter have provided most of the data for $B>19$, the total number of quasars with $B<19$ being about $10^{5}$ in agreement with the aiscussion given previously.

Related to quasars are the BL Lac objects, which are characterized by large amplitude photometric variability and by strong polarization $(5-40 \%)$. Almost nothing is known about possible radio quiet BL Lac objects. Perhaps the polarization characteristics could be used as the basis for a systematic Schmidt survey.

Galactic structure is one of the classical areas of Schmidt astronomy. The work of Micluskey and others with the Case Schmidt, that of Becker with Palomar Schmidt plates, the work at Bergedorf and elsewhere have much contributed to our knowledge about the distribution of stars (see for example Blaauw and Schmidt 1965). Interstellar absorption has caused major problems in some of these studies. The ease with which $21-\mathrm{cm}$ and other radio studies could bypass this problem has perhaps shifted the emphasis away from this type of work in the galactic plane, but it remains necessary to search for distant $O B$ stars to provide distance estimates for features identified in the radio astronomical studies.

At higher galactic latitudes, the problems of interstellar absorption diminish. Currently, the work of Becker is being extended by Sandage down to fainter magnitudes with larger telescopes. Again, it is seen here how one passes from a first view with schmidt telescopes to more detailed studies with large telescopes in small areas selected on the basis of the earlier schmidt work. Partly for galactic structure studies, but mainly to find white dwarfs and other 
"blue stars", extensive surveys of stars with small or negative U-B or $\mathrm{U}-\mathrm{V}$ colours have been made by Haro, Luyten, Richter and others with the Tonanzintla, Palomar and Tautenburg Schmidt telescopes. The change in emphasis in these surveys is noteworthy. While Luyten (1965) still could write that "unrecognized blue galaxies may also be included, but this is merely a calculated risk", at present many studies of this type are done with blue galaxies and quasars as the primary objective.

One of the fundamental problems in galactic structure is the determination of the faint end of the luminosity function. A common technique - although giving severely biased samples - is to look for objects of large proper motion on Schmidt plates. As shown by Luyten, accuracies of a few times $10^{-2}$ arc seconds per year may be obtained from repeat plates of the Palomar survey, and the publications of the Observatory of Minnesota contain numerous catalogues of high proper motion stars. Present evidence does not support the idea that low luminosity stars account for much of the "missing mass" near the sun. However, "degenerate black dwarfs", stars with too low a mass to achieve hydrogen burning, still could make a large contribution. As an example in the models of Staller and de Jong (1981), a case is described where black dwarfs account for the missing mass but where the all-sky number of $10^{5}$ is reached only at $V=23$. A proper motion survey on IV-N plates for such cool stars could be of interest.

Spectral surveys with objective prisms have led to the discovery of many interesting stars: WK, $\mathrm{Ap}, \mathrm{Am}, \mathrm{Ba}, \mathrm{S}, \mathrm{H} \alpha$ emission stars and others. In other studies, some such stars have been found by taking plates through specially chosen relatively narrow colour filters. Searches for variable stars, supernovae, etc. represent another common area of research with Schmidts.

Of particular interest in studies of galactic evolution are the first generations of stars to form. Do these have zero or very low metal abundances or were heavy elements already present at a very early stage? The answer to this question must come from Schmidt telescope searches for probably rare "zero" metal stars. An interesting attempt to find such stars is being made by Shectmann and Preston at Palomar who use a filter isolating a small spectral interval around the $\mathrm{Ca} k$ line and an objective prism. The filter cuts down on the sky background and reduces the overlap of spectra; consequently, faint magnitudes may be reached.

Schmidt telescopes are also of much use in the study of interstellar matter. Planetary nebulae have been looked for by Abell, kohoutek and others. It is interesting to note that in a list of 83 new planetaries discovered between 1978 and 1981 (kohoutek 1983), there are still 15 objects found on the Palomar survey. Apparently, even this very extensively searched survey still has many objects to be discovered. H II regions and supernova remnants are another area of Schmidt astronomy: It would be difficult to study large objects 
like the Gum nebula or the Vela Supernova kemnant with small fiela instruments. Numerous dark nebulae have also been found on the sky surveys.

Finally, in the solar system, searches for asteroids on Schmidt plates are of importance. Of special interest are the earth crossing asteroids (Apollo, Amor, etc.) which may have had a significant impact on the appearance of the surface of the earth and on the evolution of life on earth. Special searches as well as serendipitous finds have now led to the discovery of 49 such objects (Shoemaker 1983). An entirely different area of Schmidt work concerns comets, where a large field is needed for proper imaging of the long tails.

THE FUTURE OF SCHMIDT TELESCOPES

From the preceding discussion it is clear that existing schmidt telescopes still have many years of fruitful work in front of them. With regard to the longer term future, however, two issues appear to be of prime importance: detectors and a Schmidt telescope in space. With regard to the former, further improvements in photographic plates certainly would be important. The introduction of CCD-type detectors, however, could make a qualitative change. At present, CCDs are still small (few $\mathrm{cm}^{2}$ ), but there does not appear to be, in principle, any objection to fitting the curved focal place of a schmidt telescope with a mosaic of CCDs. Also the data handling problem probably would not be an obstacle a decade from now. One may wonder, however, whether pushing the Schmidt images a further 2-3 magnituaes fainter would justify the cost of such an approach. Schmidt astronomy has been particularly fruitful because until now most objects found with Schmidt telescopes could be studied in more detail and also spectroscopically with larger telescopes. This would no longer be the case in a Schmidt telescope that would go much deeper. Certainly, one can think of programmes to do at, say, $V=25$ - for example an astrometric search for very faint black dwarfs, but the number of possible programmes seems rather limited.

More promising might be a Schmidt telescope in space; the access to the uv and near IR part of the spectrum could lead to many discoveries. In addition, the potentially better angular resolution and the somewhat reduced sky brightness might allow also deeper surveys in the optical domain with a good separation between galaxies, quasars and stars; if so, this might have interest in the study of the distribution of matter in the universe on the largest scale.

The success of such a Schmidt again depends on the development of a detector that can make use of the resolution achievable in space, and which at the same time covers a large area. The balance between field and angular resolution, both from the optical and the detector point of view, will need careful consideration. 


\section{REFERENCES}

Abel1, G.: 1958, Astrophys. J. Suppl. 3, pp. 211-288

Blaauw, A. and Schmidt, M. eds.: 1965, Stars and Stellar Systems V: Galactic Structure, U. of Chicago Press

Dimitroff, G.z., Baker, J.G.: 1945, "Telescopes and Accessories", Blakiston

Kohoutek, L.: 1983, IAU Symposium 103, pp. 17-30

Lauberts, A.: 1982, The ESO/Uppsala Survey of the ESO (B) Atlas, ESO

Laustsen, S., Richter, W., van der Lans, J., West, R.M.., and

Wilson, R.N.: 1977, Astron. Astrophys. 54, pp. 639-640

Luyten, W.J.: 1965, Stars and Stellar Systems V: Galactic Structure, U. of Chicago Press, p. 394

Markarian, B.E.: 1969, Astrophys. 5, pp. 286-301

Nilson, P.: 1973, Uppsala General Catalogue of Galaxies, Uppsala Astr. Obs. Ann. 6

Osmer, P.S.: 1980, Astroph. J. Suppl. 42, pp. 523-540

Sandage, A.: 1965, Astroph. J. 141, pp. 1560-1578

Schmidt, M. and Green, R.F.: 1983, Astroph. J. 269, pp. 352-374

Shoemaker, E.M.: 1983, Ann. Rev. Earth Planet. Sci. 11, pp. 461-494

Staller, R.F. and de Jong, T.: 1981, Astron. Astrophys. 98, pp. 140-148

van Woerden, H.: 1980, ESO/ESA Workshop on Dwarf Galaxies, ESO, pp. $23-29$

Vorontsov-Velyaminov, B.A.: 1959, Atlas and Catalogue of Interacting Galaxies, Moscow State University

Vorontsov-Velyaminov, B.A. and Arhipova, V.P.: 1968, Morfologiceskij Katalog Galaktik, Moscow State University

Woltjer, L. and Setti, G.: 1982, "Astrophysical Cosmology", Pont. Acad. Scient. Scripta Varia 48, pp. 293-313

Zwicky, F.: 1971, Catalogue of Selected Compact Galaxies and of Post-Eruptive Galaxies, F. Zwicky publ.

Zwicky, F., Herzog, E., Wild, P., Karpowicz, M. and Kowal, C.T.: 1968, Catalogue of Galaxies and of Clusters of Galaxies, Cal. Inst. of Technology, Pasadena 